\title{
Teaching the PPP Network Course Based on Network Simulator
}

\author{
Anbao Wang \\ School of Computer and Information, Shanghai Second Polytechnic University, Shanghai 201209 China \\ e-mail: abwang@sspu.edu.cn
}

\begin{abstract}
Due to the complexity of network, the original teaching method cannot meet the needs of development of network. Network simulation is an effective means of researching in network protocols and a new kind of network design and optimization technique, it can provide reliable network planning design of quantitative basis, also can test plan, or compare different design schemes. This paper introduces the principle and characteristic of ns3, proposes using open source software ns3 as an assistant means to improve the teaching quality, and also discusses the application of ns3 in network education as well. An example is provided at the end of this paper.
\end{abstract}

Keywords- teaching; PPP network; ns3, network simulator;

\section{SHORTCOMING OF TEACHING IN CURRENT NETWORK COURSE}

\section{A. Shortcoming}

Modern Information Network courses are mainly related to a variety of network protocols, which teaching contents are mostly based on the five-layer reference model of TCP/IP. Due to the limited analyze for the theory of protocol in the teaching, students often feel dry and abstract in the learning process.

Most people feel it is difficult to understand in the end of the course. Most of the existing teaching means are the principle course plus the experiment course. Principle course basically adopt such teaching methods as writing on blackboard, using multimedia and so on. Multimedia mostly use PowerPoint slides, just simply move books onto the computer screen, so enhancing the role of teaching performance is little; In addition, because of the complexity of the network, school cannot buy a lot of network equipment; Furthermore, network development is fast, various new protocols and equipment are updated constantly, it is difficult to build an environment of network to practice teaching, so the experiment method cannot be used to allow students access to perceptual knowledge. Even if there is a real experiment environment, facing to huge and complex network environment, students will likewise not gain the upper hand, and are difficult to understand the real effect brought about by a variety of factors. So a lot of methods are researched to get good teaching results. [14]

\section{B. The advantages of using the network simulator}

Network simulation technology has the following characteristics [6]:

a) The entirely new simulation experiment mechanism makes it have the characteristics of gaining high reliability results under a network environment of high degree of complexity.

b) Forecast function of network simulation is unmatched by any other method.

c) Wide range of use, both optimization and expansion of the existing networks and design of the new network can be used, and particularly applicable for design and optimization of Medium and large network.

d) Low initial application cost, only very few funds will be able to provide practical network design and operating environment for large number of students, furthermore, the constructed network model can continue to use so that the latter investment will still decline continuously.

e) It is flexible, vivid and visual to use simulator for teaching. To teach via network simulator, students can visually see the dealing of the network protocol and understand the effect of various environmental or other factors on the network, can also demonstrate the advantages and disadvantages of various strategies through comparison.

f) The simulation results can be reproduced and easily analysis. In this platform, the experimenter can obtain "ideal" network environment via configuring environmental parameters and can real-time track and record important information of key node so as to gain the first-hand information about network performance evaluation. Moreover, certain special circumstances can be reproduced at any time, which is difficult to do in the real network.

\section{INTRODUCTION TO NETWORK SIMULATOR NS3}

The ns3 simulator is a discrete-event network simulator targeted primarily for research and educational use. In ns3, the simulator is written entirely in $\mathrm{C}++$, with optional Python bindings. Simulation scripts can therefore be written in $\mathrm{C}++$ or in Python. The results of some simulations can be visualized by nam, but new animators are under development. Since ns3 generates pcap packet trace files, other utilities such as Wireshark can be used to analyze traces as well [8-9].

ns3 is a free software simulation platform which aims at network technology and whose source code is open. Researchers can use it easily to develop network technology. ns3 contains an abundance of modules, almost relating to all the aspects of network technology. A large number of people are using or attempt to use ns3 in the education sector and academic community at present [1-7]. 


\section{THE APPLICATION IN TEACHING}

\section{A. The General Process for Network Simulation Using ns3}

Firstly we must analyze which layer simulation touches on. ns3 simulation is based on $\mathrm{C}^{++}$programming. Utilizing existing network elements of ns3 to achieve simulation, there is no need to amend ns3 itself, just to compile $\mathrm{c}^{++}$ script. If there is not network elements required in ns3, it needs to expand ns3 and add the necessary network elements, that is, adding a new c++ category and compiling new $\mathrm{c}^{++}$ script. Supposing that the user has completed expansion of ns3, or components containing in ns3 have met the requirements, then carrying out a step of simulation is approximately as follows: [14-15]

a) Starting to compile $\mathrm{c}^{++}$script. The first thing is to configure simulation network topology structure, at this time the basic features of chain can be determined, such as delay, bandwidth and loss strategies and so on.

b) Establishing protocol agency, including protocol binding of end equipment and establishment of communication business model.

c) Configuring parameters of business model, so as to determine the distribution of business in network.

d) Setting the trace target. ns3 saves the entire simulation process via trace files. After simulating, the user can analyze and research the trace files.

e) Compiling other assistant process, setting the time of simulation of starting and ending, then the $\mathrm{c}^{++}$script is completed.

f) Using ns3 to explain and execute $\mathrm{c}^{++}$script compiled just now.

g) Analyzing the trace files and to obtain useful data. Adjust configuring topology structure and business model, then re-simulating above process.

\section{B. The concept of the Point-to-Point Protocol (PPP)}

The Point-to-Point Protocol is designed for simple links which transport packets between two peers. These links provide full-duplex simultaneous bi-directional operation, and are assumed to deliver packets in order. It is intended that PPP provide a common solution for easy connection of a wide variety of hosts, bridges and routers [12]. PPP is comprised of three main components: [13]

1. A method for encapsulating multi-protocol datagrams.

2. A Link Control Protocol (LCP) for establishing, configuring, and testing the data-link connection.

3. A family of Network Control Protocols (NCPs) for establishing and configuring different network-layer protocols.

The Point-to-Point Protocol (PPP) is working at the second layer of the network, and it provides the service for the network layer, so when teaching the PPP protocol, Firstly, the basic theory for the PPP protocol is presented, for example, the Encapsulation, Error Control, and Transparent Transmission, they are the three key points in the MAC layer.

During the teaching period, the method for encapsulating multi-protocol datagrams is introduced. The figure 1 is to point out how the PPP encapsulates the datagram of network layer.

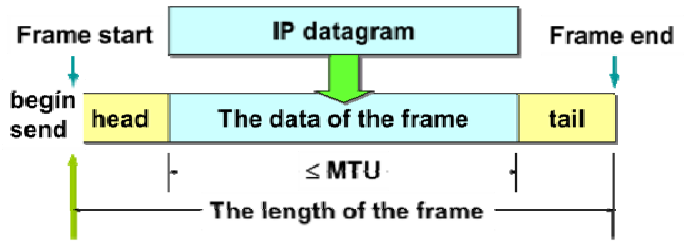

Figure 1. Example of a ONE-COLUMN figure caption.

When teaching the theory of PPP protocol, we can cut some snippet from the ns3 source code, explain the code to the students in order to help them to understand the format of the PPP frame and the working process of PPP . for example the PPP header is defined in file ppp-header.h, and the key code is displayed below.

void SetProtocol (uint16_t protocol);

uint16 t GetProtocol (void);

private:

uint16_t m_protocol;

\section{The Application of ns3 in Teaching}

A point-to-point network is built, the topology is very simple, and there is a point to point connection between node n0 and node $\mathrm{n} 1$. This simulation scripts refers to the literature [8], we simulate n0 as a client who sends package to $\mathrm{n} 1$ acting as a server using point to point connection. The parameters for the simulation at the starting time show in table1.

TABLE I. THE PARAMETERS FOR THE SIMULATION

\begin{tabular}{|l|l|}
\hline Name & value \\
\hline Device DataRate & $5 \mathrm{Mbps}$ \\
\hline Channel Delay & $2 \mathrm{~ms}$ \\
\hline Client MaxPackets & 1 \\
\hline Client Interval & $1.0 \mathrm{~s}$ (Seconds) \\
\hline Client PacketSize & 1024 \\
\hline IP address(Client) & 10.1 .1 .1 \\
\hline IP address(Server) & 10.1 .1 .2 \\
\hline Server Start & $1.0 \mathrm{~s}$ (Seconds) \\
\hline Server Stop & $10.0 \mathrm{~s}$ (Seconds) \\
\hline Client Start & $2.0 \mathrm{~s}$ (Seconds) \\
\hline Client Stop & $10.0 \mathrm{~s}$ (Seconds) \\
\hline
\end{tabular}

Device DataRate tells the PPP layer to use the value "5Mbps" as the "DataRate". Channel Delay sets the point to point channel to use the value " $2 \mathrm{~ms}$ " as the value of the transmission delay. The Client MaxPackets tells the client the maximum number of packets allowed to be sent during the simulation. Client Interval tells the client how long to wait between packets, and the Client PacketSize tells the client how large its packet payloads should be. It is told the client to send one 1024-byte packet. Server Start and Stop will cause the server to start at one second and to stop at ten seconds into the simulation. Client Start and Stop will cause the Client when to start and stop into the simulation.

Firstly we can use the function of Logging Module to get some information from the console directly.

At time 2 s client sent 1024 bytes to 10.1.1.2 port 9

At time 2.00369s server received 1024 bytes from 10.1 .1 .1 port 49153

At time 2.00369s server sent 1024 bytes to 10.1 .1 .1 port 49153 
At time 2.00737s client received 1024 bytes from 10.1.1.2 port 9

By explaining the message above, it can tell the students when and where the package is sent or received on the PPP peer layer. It can be used to explain the operation mode of the Client/Server as well.

We want to tell the students the network performance parameters in different parameters, so we change some parameters for the simulation showed in table2, the others are remains unchanged as table1.

TABLE II. THE PARAMETERS FOR THE SIMULATION

\begin{tabular}{|l|l|}
\hline \multicolumn{1}{|c|}{ Name } & \multicolumn{1}{c|}{ value } \\
\hline Device DataRate & $1 \mathrm{Mbps}$ \\
\hline Channel Delay & $1 \mathrm{~ms}$ \\
\hline Client MaxPackets & 200 \\
\hline Client Interval & $1 \mathrm{~s}$ (Seconds) \\
\hline Client PacketSize & 1024 \\
\hline Server Start & $1.0 \mathrm{~s}$ (Seconds) \\
\hline Server Stop & $100.0 \mathrm{~s}$ (Seconds) \\
\hline Client Start & $2.0 \mathrm{~s}$ (Seconds) \\
\hline Client Stop & 100.0 s (Seconds) \\
\hline
\end{tabular}

The pcap files are the output when the simulation stops, and they can be used to help the students to learn the data transfer between the two nodes between the client (node n0) and the server (node $\mathrm{n} 1$ ). When the command "tcpdump" is used to display the pcap file for node n0, the output is as follows.

reading from file ppp-0-0.pcap, link-type PPP (PPP)

2.000000 IP 10.1.1.1.49153 > 10.1.1.2.9: UDP, length 1024

2.018864 IP 10.1.1.2.9 > 10.1.1.1.49153: UDP, length 1024

3.000000 IP 10.1.1.1.49153 > 10.1.1.2.9: UDP, length 1024

3.018864 IP 10.1.1.2.9 > 10.1.1.1.49153: UDP, length 1024

4.000000 IP 10.1.1.1.49153 > 10.1.1.2.9: UDP, length 1024

4.018864 IP 10.1.1.2.9 > 10.1.1.1.49153: UDP, length 1024

5.000000 IP 10.1.1.1.49153 > 10.1.1.2.9: UDP, length 1024

The first line of the output indicates that the link type is PPP (point-to-point) which we expect. You then see the echo packet leaving node $\mathrm{n} 0$ at 2 seconds via the device associated with IP address 10.1.1.1, node n0 headed for IP address 10.1.1.2, node n1. This packet will move over the point-to-point link and be received by the point-to-point net device on node $n 1$.

The second line of the output indicates that the reply packet leaving node $\mathrm{n} 1$ at 2.018864 seconds via the device associated with IP address 10.1.1.2, node $\mathrm{n} 1$ headed for IP address 10.1.1.1 node n0. This packet will move over the point-to-point link and be received by the point-to-point net device on node $\mathrm{n} 0$.

After the simulation, the tool of Wireshark is used to display the simulation procedure; it is very useful and vivid to find how the packet is sent and received. The result for $n 0$ is display in figure 2 .

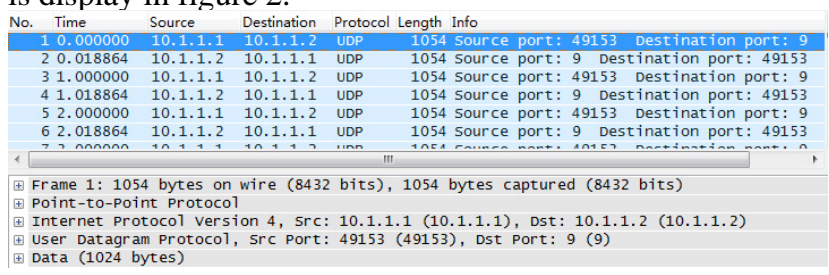

Figure 2. Procedure of the packets sent and received.
Figure 2 shows that there is a point to point connection between node n0 (ip address 10.1.1.1) and node n1 (ip address 10.1.1.2), we run this simulation about 200 seconds, the following is the analysis of the result in this experiment for node n0, and firstly, this simulation result tells the students how the Point to Point network acts step by step over time. There are 196 packages which are sent or received in n0, we can scan every packets to learn the details, for example, the first packet, the data's length is 1024 bytes, the UDP (User Datagram Protocol) is used to encapsulate the data from the upper layer in the transport layer, the Src port is 49153, and the Dst port is 9. The ipv4 is used in network layer, and then the PPP is used in second layer. We can also get the protocol hierarchy using the Wireshark which is show in Figure 3, and it is clearer than the Figure 2.

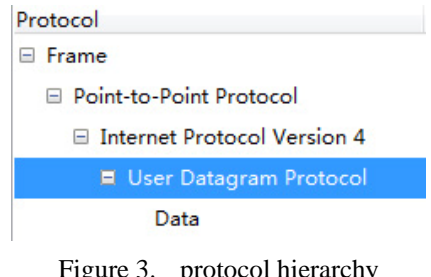

Figure 2 and Figure 3 is the simulation procedures of the selected node in this simulation, it presents packets transfer between the client and the server in a manner of easy way to understand for students, and also it is convenient to use some kind of the tools to get the statistics data of this node which is showed in Table 3.

In Table 3, it shows that in 100 seconds 196 packets were transmitted, there are 97.017 seconds between first and last packet, it is accordance with our estimate, the average packets/sec is 2.020, and the average packet size is about 1054.000 bytes. From these statistics data it provides extra help for students to master the PPP network procedure with accurate statistic information.

TABLE III. THE STATISTICS DATA IN NODE N0

\begin{tabular}{|l|l|}
\hline \multicolumn{1}{|c|}{ Name } & \multicolumn{1}{c|}{ value } \\
\hline Packets & 196 \\
\hline Between first and last packet & $97.017 \mathrm{sec}$ \\
\hline Avg. packets/sec & 2.020 \\
\hline Avg packet size & 1054.000 bytes \\
\hline Bytes & 206584 \\
\hline Avg bytes/sec & 2129.357 \\
\hline Avg Mbit/sec & 0.017 \\
\hline
\end{tabular}

From the analysis of the work procedure in the point to point network between the node $\mathrm{n} 0$ and $\mathrm{n} 1$, the students can easily understand the real process and the details of the point to point network, it can tell the student the time when the package is send or received; where the package is send or received. The size and protocol type of the packet is present to the student obviously as well.

In the rest of the experiment, one of parameters is changed in PPP network, the Client Interval is changed between $0.1 \mathrm{~s}$ to $2 \mathrm{~ms}$, and we want to tell the students how it can affect the performance in node n0 of PPP network. The result are showed in Figure 4, Figure 5, and Figure 6. 


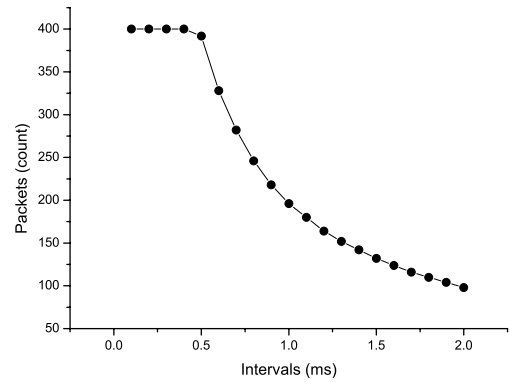

Figure 4. Packets transfer according to the Client Interval

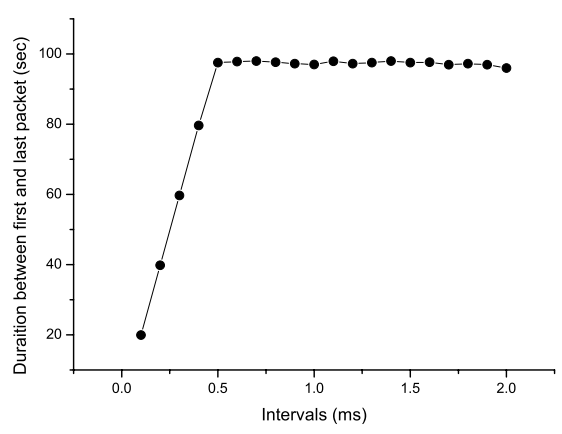

Figure 5. Duraition between first and last packet according to the Client Interval

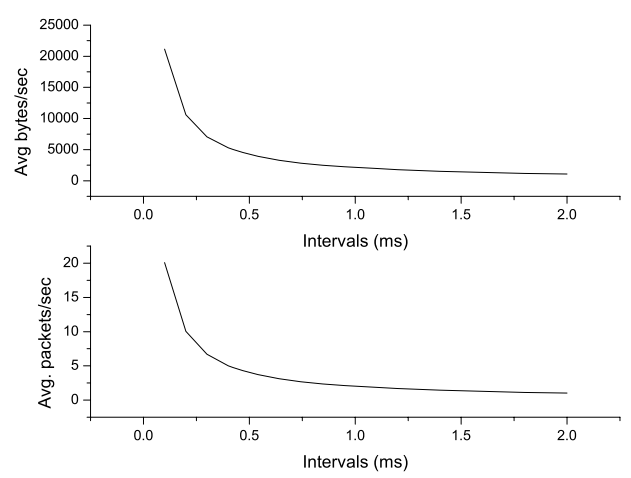

Figure 6. Avg. packets/sec and Avg bytes/sec according to the Client Interval

Figure 4 shows that packets count decreases when the client interval increases, Figure 5 shows that duration increases when the client interval increases, Figure 6 tells that the average bytes/sec and packets/sec decrease when the client interval increases. We can use Figure 4, Figure 5, and Figure 6 to help students to do some research works, to help students to learn scientific research methods and techniques.

After this experiment the students will extend the knowledge of the theory in the textbook, if students want to further understand the protocol stacks we can design the simulation environment to get more trace files, more protocol details and its construction are got by analyzing those files, the protocol working procedure can also be easily lay out lively.

\section{CONCLUSION}

Teaching practice indicates that the teaching of computer network course not only needs the content of theory teaching modernization as well as that of practical teaching in the time when the development of computer network becomes complexity more and more, but also needs to continuously explore and research advanced teaching ideas and methods and means to stimulate students' interests in this course and improve teaching quality. Here, the network simulation tool ns3 as a teaching aid to construct a virtual network simulation teaching environment, which has positive significance in improving teaching effect and level of computer network course and can make up for the shortcoming of current computer network course teaching to a certain extent. This is an entirely new exploration in the teaching of computer network course.

\section{REFERENCES}

[1] Larry L. Peterson, Bruce S. Davie. Computer Networks A Systems Approach(Second Edition) [M], Machinery Industry Press, 2001:468-482

[2] Kinsner, W., \& Pear, J. J. (1988). Computer-aided personalized system of instruction for the virtual classroom. Canadian Journal of Educational Communication, 17, 21-36.

[3] Kinsner, W., \& Pear, J. J. (1990). Dynamic educational system for the virtual campus. In Studies in technological innovation and human resources. In U. E. Gattiker (Ed.). End-user training (Vol. 2, pp. 201228). Berlin: Walter de Gruyter.

[4] R.C.T. Ellis, I. Dickinson, M. Green, M. Smith, The implementation and evaluation of an undergraduate virtual reality surveying application, in: Proceedings of the Built Environment Education Conference (BEECON 2006), September 12-13, 2006, London, UK.

[5] C.M. Li, I.C. Yeh, S.F. Cheng, T.Y. Chiang and L.C. Lien, Virtual reality learning system for digital terrain model surveying practice, Audio-Visual Education Bimonthly 48 (5) (2007), pp. 1-18 (in Chinese). Full Text via CrossRef

[6] Lin Huaiqing, Wang Bin, Qi Yuhua, Research of Teaching on Modem Information Network Curriculum Based on NS2, Ship Electronic Engineering, 2006:102-105

[7] KE Zong-wu, HONG Jia-ping, TONG Yu, CHEN Nian-sheng, DONG Wu-shi, the research of NS2-based network education, Journal of Hubei Normal University (Natural Science), 2005:65-69

[8] ns3 project ns3 Tutorial[EB/OL]. http://www.nsnam.org/, 2015-4-30

[9] ns3 project ns3 Reference Manual[EB/OL]. http://www.nsnam.org/, 2015-4-30

[10] Fall K, Varadhan K1 The NSManual[EB/OL]. http://www.isi.edu/nsnam/ns, 2015-4-30

[11] Kevin Fal, Kannan Varadhan. The ns Manual ( formerly ns Notes and Documentation) [OL]. http://www. isi. edu/nsnam/ ns/nsdocumentation.html, 2005, 11, 25

[12] Perkins, D., "Requirements for an Internet Standard Point-to-Point Protocol", RFC 1547, Carnegie Mellon University, December 1993.

[13] W. Simpson, "Request for Comments", RFC 1661, July 1994.

[14] Anbao WANG; Wenrong JIANG , Research of Teaching on Network Course Based on NS-3, First International Workshop on Education Technology and Computer Science vol 2: 629-632, 2009.

[15] Xinghui, Zhang; Anbao Wang The Study of Wireless Radio Interference Based on OPNET, 2nd International Symposium on Information Engineering and Electronic Commerce (IEEC), 1-3, 2010 . 